\title{
Solanum venturii, a suitable model system for virus-induced gene silencing studies in potato reveals StMKK6 as an important player in plant immunity
}

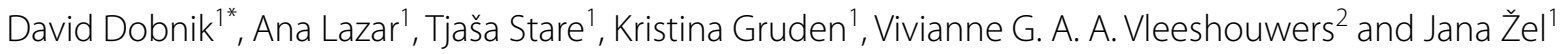

\begin{abstract}
Background: Virus-induced gene silencing (VIGS) is an optimal tool for functional analysis of genes in plants, as the viral vector spreads throughout the plant and causes reduced expression of selected gene over the whole plant. Potato (Solanum tuberosum) is one of the most important food crops, therefore studies performing functional analysis of its genes are very important. However, the majority of potato cultivars used in laboratory experimental setups are not well amenable to available VIGS systems, thus other model plants from Solanaceae family are used (usually Nicotiana benthamiana). Wild potato relatives can be a better choice for potato model, but their potential in this field was yet not fully explored. This manuscript presents the set-up of VIGS, based on Tobacco rattle virus (TRV) in wild potato relatives for functional studies in potato-virus interactions.
\end{abstract}

Results: Five different potato cultivars, usually used in our lab, did not respond to silencing of phytoene desaturase (PDS) gene with TRV-based vector. Thus screening of a large set of wild potato relatives (different Solanum species and their clones) for their susceptibility to VIGS was performed by silencing PDS gene. We identified several responsive species and further tested susceptibility of these genotypes to potato virus Y (PVY) strain NTN and N. In some species we observed that the presence of empty TRV vector restricted the movement of PVY. Fluorescently tagged PVY ${ }^{N}$-GFP spread systemically in only five of tested wild potato relatives. Based on the results, Solanum venturii (VNT366-2) was selected as the most suitable system for functional analysis of genes involved in potato-PVY interaction. The system was tested by silencing two different plant immune signalling-related kinases, StWIPK and StMKK6. Silencing of StMKK6 enabled faster spreading of the virus throughout the plant, while silencing of WIPK had no effect on spreading of the virus.

Conclusions: The system employing S. venturii (VNT366-2) and PVY -GFP is a suitable method for fast and simple functional analysis of genes involved in potato-PVY interactions. Additionally, a set of identified VIGS responsive species of wild potato relatives could serve as a tool for general studies of potato gene function.

Keywords: Potato, Virus-induced gene silencing, VIGS, Potato virus Y, PVY, Solanum venturii, StWIPK, StMKK6, TRV

\section{Background}

Cultivated potato (Solanum tuberosum L.) is, after rice, maize and wheat, the world's fourth most important food crop (http://faostat.fao.org/). Its susceptibility to

\footnotetext{
*Correspondence: david.dobnik@nib.si

${ }^{1}$ Department of Biotechnology and Systems Biology, National Institute of Biology, Večna Pot 111, 1000 Ljubljana, Slovenia

Full list of author information is available at the end of the article
}

wide range of pathogens, which diminish its yield, could therefore have a great impact in the food production chain. One of the most important potato pathogens is the potato virus $Y(\mathrm{PVY})$. The necrotic isolates of $\mathrm{PVY}$ are still responsible for huge agronomic and economic losses [1]. The ability of viruses to cause a disease is determined at the level of molecular interactions between the host plant counterparts and virus factors that can lead to 
compatible (sensitive) or incompatible (resistant) interactions [2]. The differential sensitivity of potato cultivars lies in their different genetic background, where the resistant ones usually possess $R y$ (extreme resistance) or $N y$ (hypersensitive resistance) gene (reviewed in [3]). However, recent transcriptomic studies revealed the complexity of signalling network involved in the defence response of potato against PVY [4-8].

The use of transient transformation would be a welcome additional tool to evaluate the role of individual genes in potato. Stable genetic transformation is a less preferred approach, since it is a lengthy process, taking at least 6 months to produce substantial number of transformed plants that can be used for functional experiments. However, to study the interaction between potato and PVY, the gene expression must be modified throughout the whole plant and not only locally, as the virus also spreads systemically. Virus-induced gene silencing (VIGS) is an optimal tool for this purpose, as the virus vector spreads throughout the plant and causes reduction in activity of selected gene in the whole plant based on post-transcriptional gene silencing (PTGS) [9].

Until now, several VIGS vectors originating from RNA and DNA viruses were developed $[10,11]$. The Tobacco rattle virus (TRV) vector is the most widely used due to its wide host range and mild symptoms [12]. Some VIGS studies with potato using TRV vector have been already described [13, 14], however they are not being used as routinely in potato as for example in model plant species Nicotiana benthamiana.

Tuber-bearing Solanum species that belong to section Petota, represent a large pool of potato relatives, potentially suitable as model species. Section Petota contains mostly species from North and South Americas [15, 16], e.g. Solanum bulbocastanum, Solanum stoloniferum and also the cultivated potato Solanum tuberosum. The resource was already used by the potato breeders to introduce the desired traits into cultivars [17]. As these are the closest relatives to cultivated potato, they would serve as a good model system with best possible data translation to cultivated potato cultivars. Large database was already constructed with information on phylogeny [18] and Phytophthora infestans resistance in wild potato relatives [19], but no information exist on resistance or susceptibility of these species to viruses.

In order to evaluate the potential application of TRVbased VIGS for functional analysis of potato genes we first screened cultivars for their susceptibility to TRV-based VIGS. As none of the cultivars responded to VIGS, we decided to test wild potato relatives as the closest possible model system, which could be used for potato-PVY interaction studies. We screened an assortment of wild potato relatives for their susceptibility to
TRV-based VIGS and selected the most responsive species for further evaluation of their response to PVY infection. Finally, we were able to select good candidates for studies of gene function in potato-PVY interaction. To show that the selected system is applicable for functional evaluation of a potato genes, we selected a mitogen-activated protein kinase (MAPK) gene StWIPK (A. thaliana orthologue AtMAPK3) and a mitogen-activated protein kinase kinase (MKK), gene StMKK6. In several studies the members of WIPK family were shown to be involved in wound and pathogen responses [20-24], response to oxidative stress $[25,26]$, drought response and stomata development [27-30]. WIPK was shown to be responsive after the infection of $N$. benthamiana with potato virus $\mathrm{X}$ (PVX) and PVY [31]. MKK genes were also shown to be involved in regulation of cytokinesis [32-35] in abscisic and salicylic acid signalling [36, 37], in salt stress response [36] and also in response to pathogen infection [37-40]. With the developed methodology, we showed that downregulation (silencing) of StMKK6 promotes the viral spread, whereas silencing of StWIPK did not affect the virus.

\section{Results and discussion \\ VIGS on different potato cultivars}

TRV-based VIGS is a wide-spread system for gene silencing, therefore our aim was to check its applicability on all the genotypes we use in PVY-potato interaction studies in our lab (Igor, PW363, Santé, Rywal, NahG-Rywal, Désirée, Désirée Glu-III and NahG-Désirée). They are differently sensitive to PVY (Additional file 1) because of their genetic background. NahG plants were genetically modified to impair salicylic acid (SA) accumulation $[7,41]$ and Glu-III transgenic Désirée harbours $\beta-1,3-$ glucanase class III gene under control of $35 \mathrm{~S}$ promoter [42]. No literature data existed on the susceptibility of the selected cultivars to TRV-based VIGS. Experimental plants were agroinfiltrated with a mixture of pTRV1 and pTRV2:CaPDS, constructs for silencing phytoene desaturase (PDS). When silenced the carotenoid biosynthesis is supressed, which makes plants susceptible to photobleaching [43] that can easily be visualized. $N$. benthamiana plants were used as positive control and after 12 days post agroinfiltration, plants have already started to show the photo-bleaching phenotype, which persisted throughout the experiment. To show that the original pTRV2:CaPDS construct originating from pepper, would work also on other plant species, we have analysed the sequence identity of different PDS genes. The observed nucleotide sequence identity of Capsicum annuum PDS was higher than $95 \%$ when compared to S. tuberosum or S. nigrum PDS and $92.7 \%$ when compared to $N$. benthamiana PDS (Additional file 2). As $N$. 
benthamiana plants served as positive control and were always showing photo-bleached phenotype, we concluded that pTRV2:CaPDS should also work on Solanum species.

Both NahG-Rywal and NahG-Désirée plants developed strong disease symptoms (leaves have dried up and fallen off) already at 10 days post agroinfiltration. Most probably the lack of SA allowed high TRV accumulation, which was devastating for the small plants. None of other cultivars showed any signs of photobleaching. Cultivar Igor responded by dropping the infiltrated leaves, what often occurs after PVY inoculation in this cultivar [44], potentially indicating the multiplication of TRV. To our knowledge, there are only two publications presenting data on VIGS in potato using TRV, showing only three cultivars of $S$. tuberosum (GT12297-4, Cara and Pentland Ivory) as susceptible $[13,14]$. Noteworthy, Brigneti et al. had applied VIGS to potato plants grown from seeds [13], a system not used in many of the labs, as the propagation of potatoes in tissue culture is required for retaining the desired genotype.

\section{VIGS and wild potato relatives}

Brigneti et al. showed that two wild potato relatives, $S$. bulbocastanum and S. okade, were susceptible to VIGS [13]. As S. tuberosum cultivars tested in our experiments were not responsive to TRV-based VIGS, we have therefore decided to find a set of VIGS susceptible wild potato relatives that could serve as model plants for studies of potato genes. We have tested 73 different clones representing 34 different species of wild potato relatives (Additional file 3), from which all were already tested for resistance/susceptibility to P. infestans [19]. Decision to include several clones of the same species was based on observed different resistance level against $P$. infestans. An online phylogenetic analysis to illustrate the relation between $S$. tuberosum and wild potato relatives was conducted within SolRgene [19] with clones that are available in database (Fig. 1). All tested plants were agroinfiltrated with a mixture of pTRV1 and pTRV2:CaPDS and photo-bleaching symptoms were scored 21-24 days post agroinfiltration (dpa). The extent of the recorded photo-bleaching phenotype was classified into four groups: (1) moderate to strong silencing, (2) moderate silencing, (3) low-level silencing and (4) no silencing. 15 clones (10 species) have shown moderate to strong silencing, 4 clones ( 4 species) have shown moderate silencing and 10 clones (10 species) have shown lowlevel silencing (Fig. 1). 44 clones (18 species) have shown no silencing (Additional file 3). As the results suggest, the pool of wild potato relatives is not amenable to TRVbased VIGS, with more than half of tested clones being nonresponsive. Even with the overall highly responsive species like Solanum venturii or S. stoloniferum, there were still some non-responding clones indicating that subtle differences in genotype or epigenetic differences can affect the outcome of plant-TRV interaction. Our results also confirm previous data [13], as S. bulbocastanum and $S$. okade species were shown to be in the most responsive group, even though we performed our tests on the plants propagated in stem tissue cultures in contrast to the seed grown plants. When integrating this data with the phylogenetic analysis we observed that at least half of the most responsive clones (mostly $S$. venturii) were relatively closely related to $S$. tuberosum (Fig. 1), when referring to distance and position in a phylogenetic tree in comparison to S. tuberosum and S. lycopersicon.

\section{Susceptibility of selected wild potato relatives to PVY NTN}

For the next experiment we selected all 15 clones that were classified as strongly responsive to TRV-based VIGS (Fig. 1a). In order to use the wild potato relatives in functional analysis of genes involved in potato-PVY interaction, their response to PVY must be known. Therefore, the selected clones were inoculated with highly aggressive PVY strain NTN (PVY ${ }^{\mathrm{NTN}}$ ) and we followed its spreading to upper non-inoculated leaves. Samples were collected 14 days post inoculation (dpi), since this is the time when PVY is expected to be present throughout the plant in the case of tolerant interaction [4]. The relative viral RNA content measured in the samples is presented in Fig. 2. All of the tested clones have shown the tolerant phenotype as viral RNA was detected in upper, non-inoculated leaves in all of the clones while no symptoms of viral infection were observed (Additional file 4). S. bulbocastanum (BLB 331-2) had the lowest viral RNA content, however we were able to get the data only from 1 out of 3 plants, as the RNA isolation from other two samples was not successful (data not shown). S jamesii (JAM 355-1) and S. lesteri (LES 358-4) had also relatively low viral content, nevertheless the same low content was observed in individual plants of other clones (Fig. 2) indicating highly variable relative $P V Y^{\mathrm{NTN}}$ RNA content between plants and genotypes. When observing only the spreading of PVY, the viral content itself is not the true marker for the susceptibility as more susceptible plants don't necessarily have higher viral content [4]. We showed that there were no significant differences in the amount of viral RNA between clones when infected only with PVY ${ }^{\mathrm{NTN}}$ (Additional file 5). Most probably, the variability in the relative amount of $\mathrm{PVY}^{\mathrm{NTN}}$ RNA is indicating that the time after infection when the virus reaches the upper leaves differs between the individual plants of the same genotype, which was also observed in other experiments studying potato-PVY interaction [4]. These results will be added 

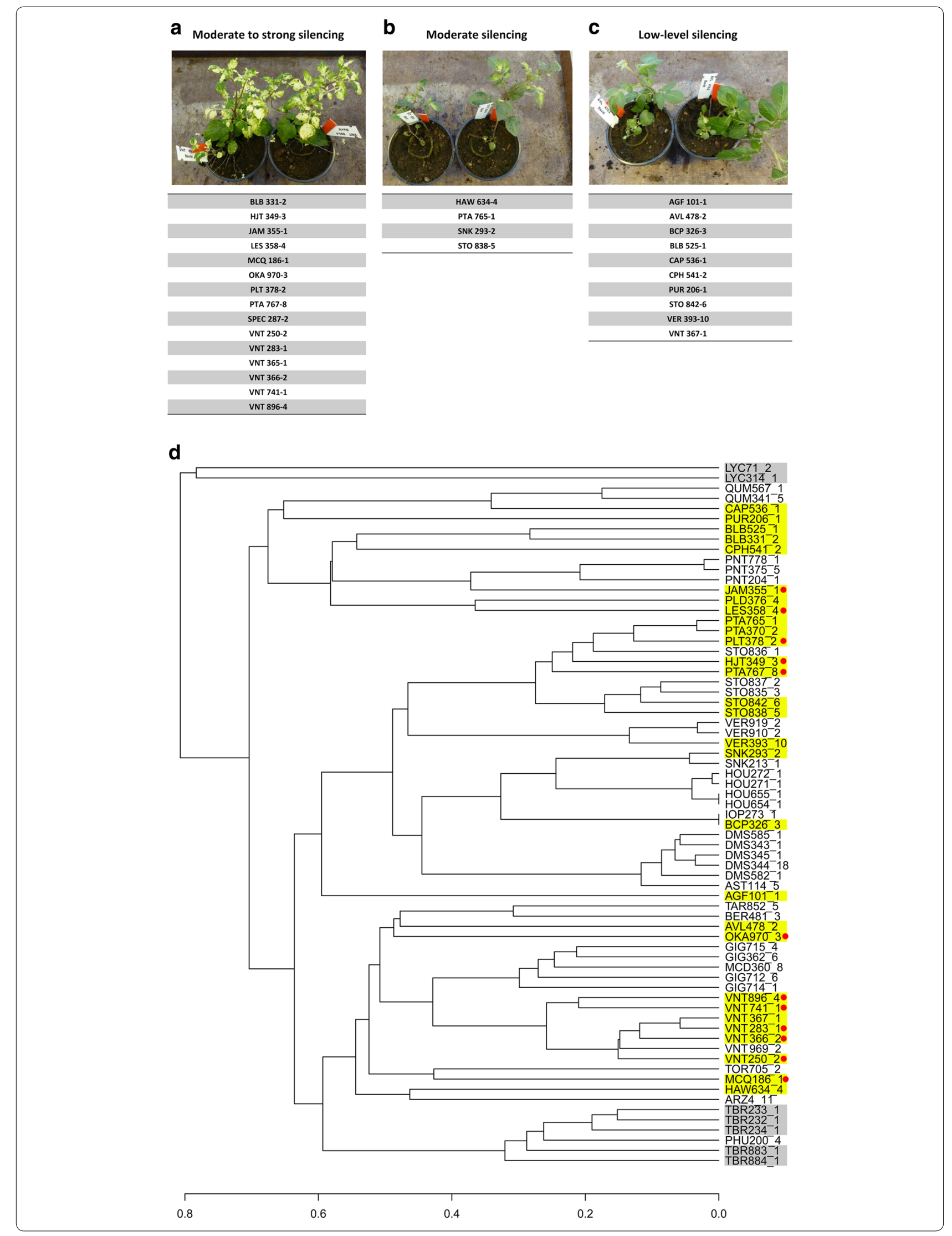

C Low-level silencing 
(See figure on previous page.)

Fig. 1 Efficiency of TRV-based VIGS in different wild relatives of potato. The clones that responded to VIGS with TRV (silencing of PDS), were classified into three groups: a moderate to strong silencing, $\mathbf{b}$ moderate silencing and $\mathbf{c}$ low-level silencing. Photograph at the top of the panels $\mathbf{a}-\mathbf{c}$ shows silencing phenotype representative for each group. Responsive clones are listed in each corresponding panel. Phylogenetic tree of 62 clones (d) that were available in SolRgene database was constructed with interactive online tool using UPGMA method. All VIGS responsive clones are highlighted in yellow. The clones from the group of moderate to strong silencing are labelled with a red dot. Solanum lycopersicum (LYC) and Solanum tuberosum (TBR) clones (highlighted in grey) were added to the phylogenetic tree to illustrate the relations of wild potato relatives to tomato and cultivated potato. AGF, Solanum agrimonifolium; AVL, Solanum avilesii; BCP, Solanum brachycarpum; BLB, Solanum bulbocastanum; CAP, Solanum capsicibaccatum; CPH, Solanum cardiophyllum; HAW, Solanum hawkesianum; HJT, Solanum hjertingii; JAM, Solanum jamesii; LES, Solanum lesteri; MCQ, Solanum mochiquense; OKA, Solanum okadae; PLT, Solanum polytrichon; PTA, Solanum papita; PUR, Solanum piurana; SNK, Solanum schenckii; SPEC, Solanum species; STO, Solanum stoloniferum; VER, Solanum verrucosum; VNT, Solanum venturii

to the SolRgene database and will complement the information on susceptibility to $P$. infestans in SolRgene database [19].

\section{The effect of TRV infection on PVY ${ }^{\mathrm{NTN}}$ spread in selected wild potato relatives}

We showed that PVY ${ }^{\mathrm{NTN}}$ spreads in all of the selected clones and in order to use these clones in VIGS studies, we analysed the effect of empty TRV VIGS vector on spreading of PVY ${ }^{\mathrm{NTN}}$. As when performing the normal VIGS experiment, we have agroinfiltrated 1 week old plants with empty TRV vector and after 3 additional weeks inoculated the plants with PVY ${ }^{\mathrm{NTN}}$. Again, we have tested for presence of PVY ${ }^{\mathrm{NTN}}$ RNA in the samples of non-inoculated leaves collected 14 days after $\mathrm{PVY}^{\mathrm{NTN}}$ inoculation. Although we detected significant differences in PVY ${ }^{\mathrm{NTN}}$ content in limited number of cases (Additional file 5), as already discussed above, rather than the relative viral RNA content, the more important indicator of susceptibility to viral infection is the number of plants with detected PVY in upper leaves [42]. In our experiments, prior agroinfiltration with empty TRV vector for VIGS reduced the number of plants successfully infected with $\mathrm{PVY}^{\mathrm{NTN}}$ (Fig. 2). In three (BLB 331-2, SPEC 287-2 and VNT 741-1) out of seven clones where the reduction in number of infected plants was observed (BLB 331-2, HJT 349-3, MCQ1861, SPEC 287-2, VNT283-1, VNT 365-1 and VNT 741-1), only one out of five plants was systemically infected.

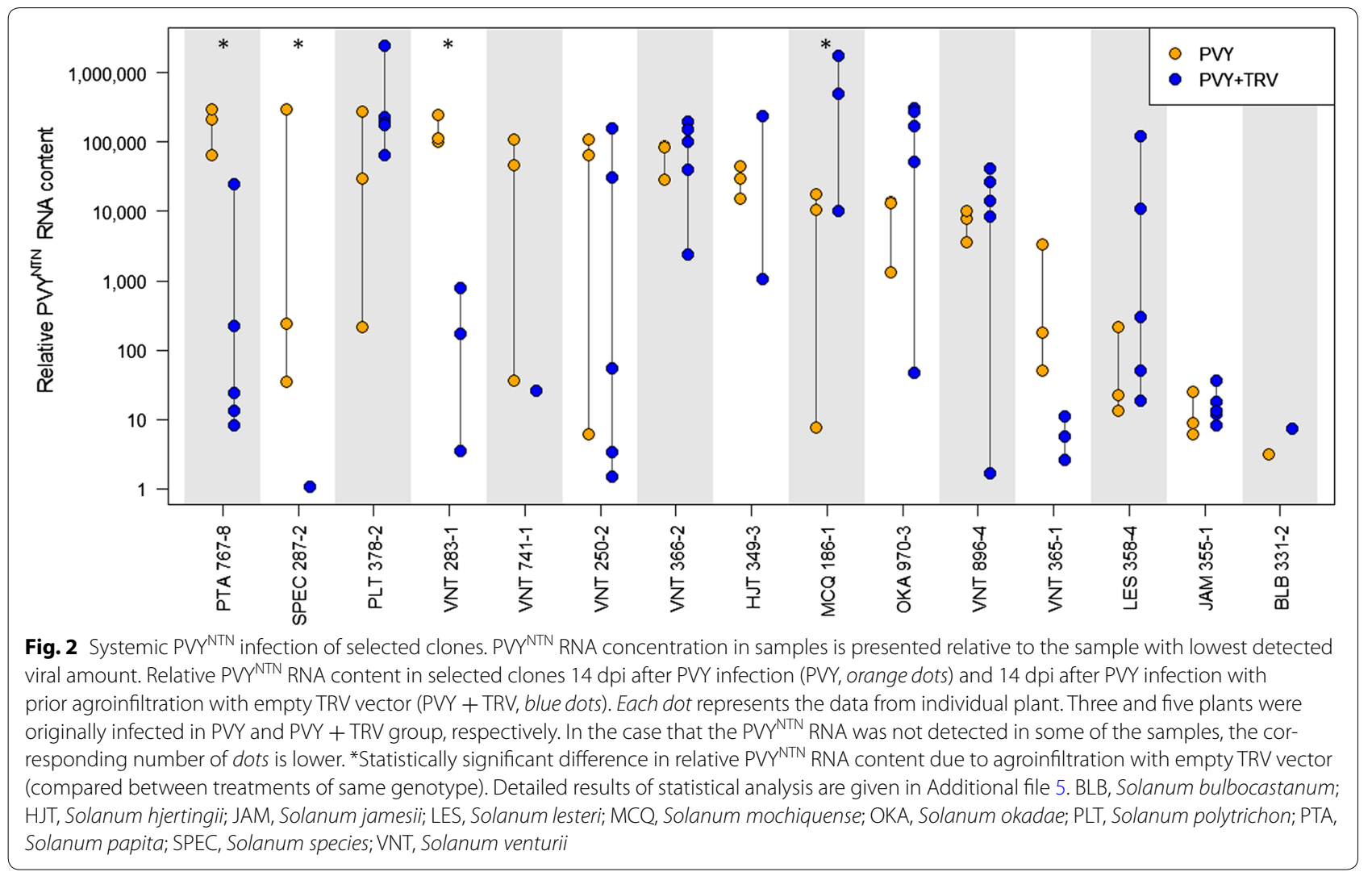


For functional analysis studies there should be no effect of TRV on the spreading of PVY, therefore we also compared the data of PVY ${ }^{\mathrm{NTN}}$ content measured in both experiments (with and without prior TRV agroinfiltration) (Fig. 2, Additional file 5). We showed that the clones suitable for further studies with VIGS, in which the TRV did not affect the spreading of $\mathrm{PVY}^{\mathrm{NTN}}$, are $S$. jamesii (355-1), S. lesteri (358-4), S. okade (970-3), S. polytrichon (378-2) and S. venturii (250-2, 366-2 and 896-4). S. bulbocastanum, which was previously already used in TRVbased VIGS experiments for testing resistance genes against $P$. infestans [13], was shown not to be suitable for VIGS in the case of PVY studies, due to the influence of TRV on the course of PVY infection (Fig. 2).

\section{Susceptibility of selected wild potato relatives to PVY ${ }^{\mathrm{N}}$-GFP} Parallel to these studies, an infectious clone of $\mathrm{PVY}^{\mathrm{N}}$ strain fluorescently labelled with green fluorescent protein (GFP) was developed in our laboratory [45]. Detailed spatio-temporal analyses of viral multiplication are only feasible using fluorescently tagged viruses as then the virus can be monitored in a non-invasive manner using confocal microscopy. In the course of testing the developed infectious clone, also its ability to infect the wild potato relatives was analysed [45]. The green fluorescence, a trace of PVY ${ }^{\mathrm{N}}$-GFP multiplication, was detected in a set of TRV-based VIGS responsive wild potato relatives in inoculated leaves (at $14 \mathrm{dpi}$ ), indicating the virus was successfully multiplying. On the other hand, GFP fluorescence in non-inoculated leaves was detected at 14 dpi only in five clones (MCQ 186-1, PTA 767-8, VNT 283-1, VNT 365-1 and VNT366-2) [45]. When comparing this report to our results, it is obvious, that the spreading of PVY ${ }^{\mathrm{N}}$-GFP is not as efficient as that of PVYNTN, since $P V Y^{\mathrm{N}}$ is less aggressive strain of the PVY [6]. Based on these observations we have performed a more detailed study of PVY ${ }^{\mathrm{N}}$-GFP spread in wild potato relatives. When we evaluated the GFP fluorescence in noninoculated leaves of wild potato relatives at $14 \mathrm{dpi}$, only VNT 366-2 and VNT283-1 showed the whole area of the non-inoculated leaves covered in green fluorescence and in other three (MCQ 186-1, PTA 767-8 and VNT 365-1) only local patches with green fluorescence were detected. The example of green fluorescence signal in the leaves of VNT 366-2 can be seen in Fig. 3. It is important to mention that some structures exhibiting green fluorescence, most probably due to accumulation of secondary metabolites, can be detected in mock inoculated leaves (Fig. 3), however the green fluorescence pattern can be clearly distinguished from true positive signal (Fig. 3). Finally, VNT 366-2 and VNT 283-1 would be the most suitable candidates for VIGS test subjects, but because in VNT 283-1 the TRV infection interfered with the speed of PVY movement (Fig. 2), we concluded that VNT 366-2 is the most suitable clone for experiments including TRV-based VIGS and PVY ${ }^{\mathrm{N}}$-GFP for functional analysis of genes involved in potato-PVY interaction. However, we have also observed that the PVY ${ }^{\mathrm{N}}$-GFP was spreading in VNT 366-2 agroinfiltrated with empty TRV with slower rate. The green fluorescence of PVY ${ }^{\mathrm{N}}-$ GFP in the non-inoculated leaves of an empty vector agroinfiltrated control plants was usually detected at $21 \mathrm{dpi}$ or later (in individual plants green fluorescence was detected at 14 dpi, but never in all biological replicates). Nevertheless, the VIGS system with clone VNT 366-2 is applicable for functional studies of potato genes, even if using milder viral strains.

\section{Functional confirmation of StMKK6 role as positive regulator of plant defence against PVY}

To show the suitability of the selected wild potato relative (clone VNT366-2) for the functional analysis of genes involved in potato-PVY interaction, two different genes from the immune signalling cascade, kinases StWIPK and StMKK6, were analysed with this system. MAPKs are crucial in plant immune response, therefore disrupting MAPK signalling, by silencing its components, could lead to disturbed plant response to infection with PVY. Both selected genes have been previously reported as important components of plant response to viral infection [31, 37]. We have studied the effect of StWIPK and StMKK6 silencing through observing the GFP fluorescence in the upper, non-inoculated leaves, as a marker of viral spread. Silencing of StWIPK had no significant effect on the viral spread in any of the observed time points. The spread of PVY ${ }^{\mathrm{N}}$-GFP was also observed in the silenced plants and was comparable to the non-silenced ones (Table 1). There are however several reports on WIPK as a positive regulator of immune response [20, 22, 24, 31, 38, 46-49], moreover, in tobacco, silencing of $\mathrm{NtWIPK}$ led to reduced resistance to TMV $[38,50]$. On the other hand, silencing of StMKK6 showed that the kinase has important role in viral spread. Emergence of PVY ${ }^{\mathrm{N}}$-GFP multiplication marker in non-inoculated leaves of silenced plants preceded the emergence of $\mathrm{PVY}^{\mathrm{N}}$-GFP in empty vector-treated control plants. Additionally, we detected PVY in StMKK6-silenced plants more frequently than in control plants, suggesting that silencing of StMKK6 renders plants more susceptible to PVY infection (Fig. 4). These results support our recent findings that StMKK6 is involved in potato immune response against PVY and acts as positive regulator of potato defence response [37]. It has been shown that $M K K 6$ gene expression in potato is induced after PVY treatment in a SA-dependant manner [37]. SA is known to influence the expression of defence-related genes $[4,7]$, especially in response to viral 


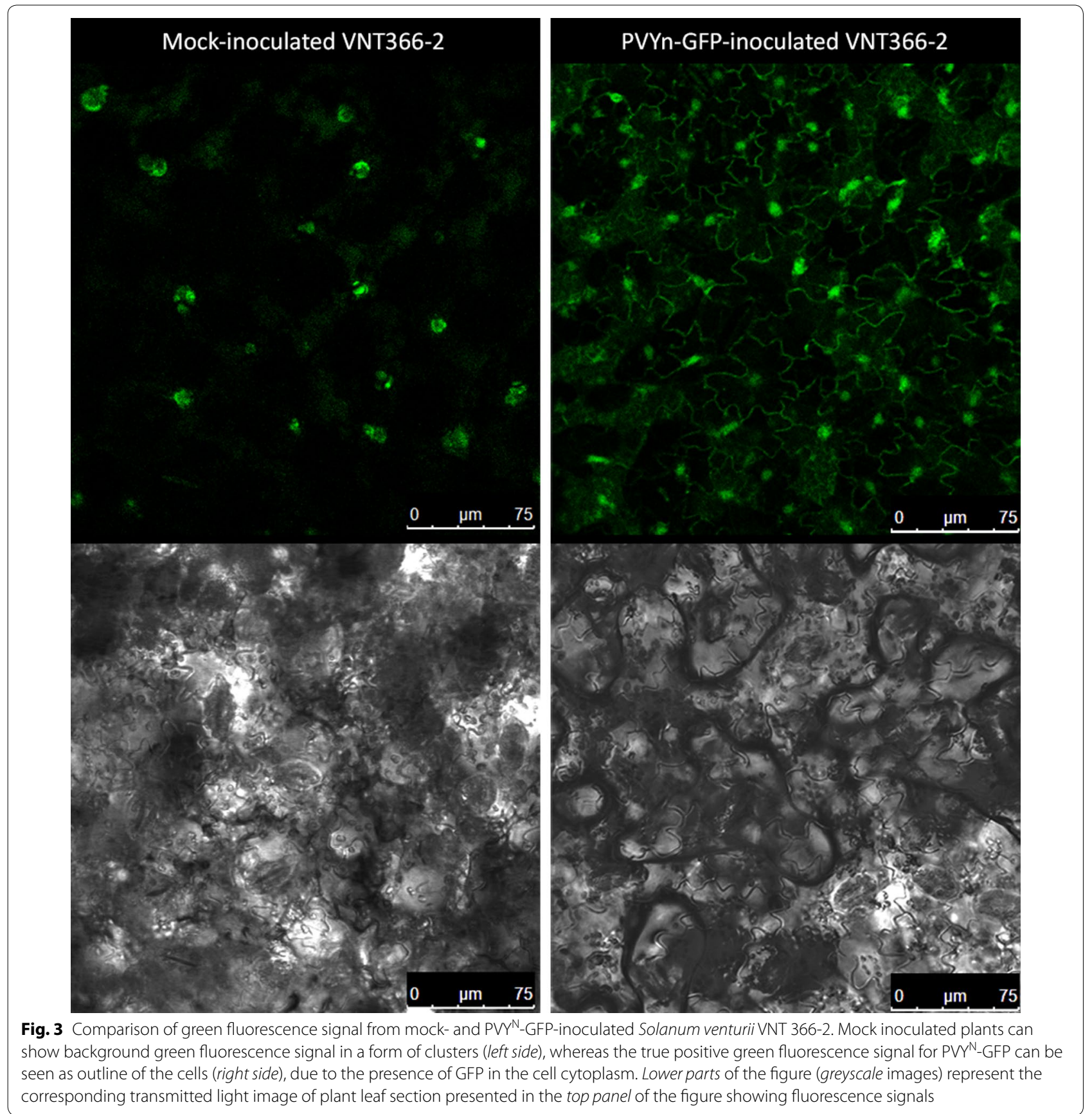

pathogens, through a finely tuned signalling cascades, where many aspects of the regulation still remain undefined [51]. However, based on the results from Arabidopsis, SA signalling regulation with kinases is explained by negative feedback loop, where SA accumulation is negatively regulated by the MAP kinase MPK4 via PAD4 and EDS1 $[52,53]$. It was shown that potato StMKK6 interacts with StMAPK4 probably through phosphorylation of StMAPK4 and additionally that StMKK6 is involved in $\mathrm{HR}$ of potato against PVY a process which is highly dependent on SA [37]. Our results of VIGS, together with our previous findings [37], therefore imply that StMKK6 is involved in potato immune signalling cascade via interaction with genes involved in SA signalling. Until now, only few reports are available on the topic of MKK6 silencing. It was shown that silencing of its orthologue has an effect on plant immune response by attenuating the resistance against TMV in $N$. tabacum, most 
Table 1 Effect of StWIPK silencing on spreading of PVY ${ }^{\mathrm{N}}$ GFP to upper non-inoculated leaves

\begin{tabular}{llll}
\hline dpi & \multicolumn{3}{l}{ Systemically infected plants (\%) } \\
\cline { 2 - 4 } & WIPK silenced & Empty vector & Positive control \\
\hline 14 & 0 & 14.3 & 33.3 \\
18 & 0 & 14.3 & nd \\
22 & 85.7 & 85.7 & 100 \\
\hline
\end{tabular}

dpi, days post inoculation with PVY ${ }^{N}$-GFP; WIPK silenced, six plants were agroinfiltrated with TRV construct for silencing WIPK gene and infected with PVYN-GFP; Empty vector, six plants were agroinfiltrated with empty TRV construct and infected with PVY ${ }^{N}$-GFP; Positive control, three plants were infected with PVY ${ }^{\mathrm{N}}$-GFP at the same time as WIPK silenced and empty vector plants; nd, not determined at this time point

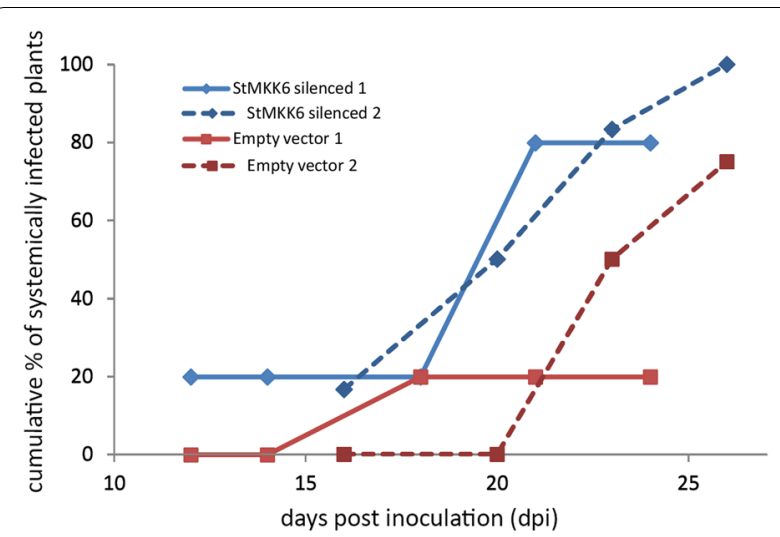

Fig. 4 Silencing of StMKK6 is increasing the speed of viral spread. Cumulative percentage of systemically infected plants after TRVbased VIGS in relation to days post inoculation is shown. The results of two independent time series experiments are presented. In first experiment a total of five biological replicates (plants) was used for each treatment; StMKK6 silenced (blue solid line) and empty TRV vector agroinfiltrated (red solid line). In second experiment a total of six biological replicates were used for StMKK6 silenced group (blue dashed line) and four biological replicates for empty TRV vector agroinfiltrated plants (red dashed line). At each time point only two or three replicates were analysed

probably through reduced activation of different transcription factors [39]. Moreover, silencing of the orthologue $\mathrm{NbMEK1}$ prevented the hypersensitive response (HR) that is triggered via NTF6 cascade [54]. The results of silenced StMKK6 reported here offer an additional functional confirmation of StMKK6 as a positive regulator of plant defence against PVY and present additional piece of evidence for the above mentioned connection of SA and StMKK6.

\section{Conclusions}

We established a fast screening system for evaluation of potato gene function, in particular in response to infection with PVY. As the cultivable potato is not suitable for
TRV-based VIGS, we have performed an evaluation of suitability of wild potato relatives for functional analysis of potato genes involved in potato-virus interaction using TRV-based VIGS on a wide set of different species. The most responsive species were further tested for susceptibility to two different PVY strains in order to select the best model species. All tested wild potato relatives were shown to be tolerant to PVY, suggesting they do not possess any resistance genes against this virus. As presented in the manuscript, the system employing $S$. venturii (VNT366-2) and $\mathrm{PVY}^{\mathrm{N}}$-GFP is the best choice for fast functional analysis of genes. Using the developed system, we have shown that StMKK6 has a role of positive regulator in potato defence against PVY. Additionally, a set of identified TRV-based VIGS responsive species could also serve as a general tool for functional analyses.

\section{Methods}

\section{Plant materials and growth conditions}

Set of wild potato relatives (wild Solanum species, Additional file 3) used in the studies is part of collection of WUR Plant Breeding, Wageningen University and Research Centre, The Netherlands. Different Solanum tuberosum cultivars (Additional file 1) are part of the collection of National Institute of Biology, Slovenia. Nicotiana benthamiana was used in VIGS experiments as control of silencing. $N$. benthamiana plants were grown from seeds and kept in a growth chamber under controlled conditions $(16 \mathrm{~h}$ light $/ 8 \mathrm{~h}$ dark cycle at $22 / 20{ }^{\circ} \mathrm{C}$ respectively). In vitro potato plantlets of different Solanum species were propagated in sterile culture boxes containing MS medium supplemented with $3 \%$ sucrose and $0.8 \%$ agar and grown in a growth chamber under controlled conditions ( $16 \mathrm{~h}$ light/8 h dark cycle at $21 / 19{ }^{\circ} \mathrm{C}$ respectively). Two-week-old plantlets were transplanted into soil and moved to a greenhouse with $22 / 20{ }^{\circ} \mathrm{C}$ day/night temperature regime.

\section{Plasmid constructs and transformation of Agrobacterium tumefaciens}

The basic set of TRV VIGS vectors used in the studies was previously described [55]. For PDS silencing we used the pTRV2:CaPDS that is based on pYL156 plasmid backbone with inserted 371-bp fragment (610-980 bp) of PDS gene from Capsicum annuum (GenBank accession X68058).

The full-length sequence of StWIPK was amplified from S. tuberosum cv. Rywal cDNA with the following primers: forward 5-ATGGTTGATGCTAATATGGGT-3 and reverse 5-GCACACAAGCTAGCACGAAC-3. The fragments were inserted into the PJET 1.2 blunt cloning 
vector (Thermo Scientific) and sequenced (GATC Biotech). For StWIPK silencing the 520-bp fragment (187$706 \mathrm{bp}$ ) was amplified from pJET plasmid harbouring StWIPK gene (GeneBank accession no. KP033231.1) with forward (5'-GAATTCTGAATGAGATGGTTG CAGTT-3') and reverse (5'-TAAGCTCCATGAAGATG CAA-3') primer. For StMKK6 silencing the 453-bp fragment (157-609 bp) was amplified from plasmid harbouring StMKK6 gene [37] (GeneBank accession no. KF837127.1) with forward (5'-GAATTCTGCCCTCAG AAACTAAGGAG- $3^{\prime}$ ) and reverse $\left(5^{\prime}\right.$-TCCTTTGTGG TTCACTAGCA-3') primer. In both cases forward primer harboured the EcoRI restriction site. The amplified fragments were inserted into the PJET 1.2 blunt cloning vector (Thermo Scientific) and sequenced (GATC Biotech). pJET plasmids harbouring St WIPK or StMKK6 fragment and empty pTRV2 plasmids were restricted with EcoRI (Gibco) and XbaI (Gibco) restriction enzymes and purified from agarose gel with Wizard SV Gel and PCR Clean-Up System (Promega). Purified fragments were ligated into restricted pTRV2 with T4 DNA ligase (Fermentas). Resulting plasmids pTRV2:WIPK and pTRV2:MKK6 were sequenced (GATC Biotech) and introduced into Agrobacterium tumefaciens strain GV3101 by electroporation (Eppendorf Electroporator 2510) following manufacturer's procedure with voltage set to $2000 \mathrm{~V}$.

\section{Bioinformatic analysis}

Phylogenetic tree of potato and its relatives was prepared with online Interactive phylogeny tool within SolRgene database (http://www.plantbreeding.wur.nl/SolRgenes/ Phylogeny/species_select.php). In first step all the species were selected and in the second step the accessions used in our experiments were selected. 11 clones out of 73 , which were used in our study for responsiveness to TRV silencing, were not available in the database and are therefore not included in the tree. Additionaly two $S$. lycopersicum and six S. tuberosum accessions were added to the phylogenetic tree to illustrate the relations of wild potato relatives to tomato and cultivated potato. A dendrogram [average linkage (UPGMA)] was created.

To check in silico whether the CaPDS construct (part of the sequence with GenBank accession number X68058.1) would have an effect on Solanum species, we have aligned different PDS sequences and checked the identity in comparison to CaPDS. The parts of sequences of PDS genes that were used were from S. tuberosum (GenBank accession number AY484445.1), S. nigrum (GenBank accession number EU434622.1) and $N$. benthamiana (GenBank accession number EU165355.1). Alignment was prepared and analysed with AlignX software (a component of Vector NTI Suite 9.0.0).

\section{Agroinfiltration for TRV-based VIGS and inoculations with PVY}

Plants, after being grown in soil for 7-10 days, were treated by co-infiltration of Agrobacterium tumefaciens strain GV3101 carrying pTRV1 and the various pTRV2 recombinants, in a 1:1 ratio as described by Du et al. [56]. pTRV2:CaPDS and empty pTRV2 plasmids were used in initial experiments and later on as controls. For testing VIGS susceptibility of different potato cultivars three plants were tested for each cultivar and for wild potato species two plants were tested for each species.

Five plants of the wild potato relatives were inoculated with buffered suspension of PVY ${ }^{\mathrm{NTN}}$ (isolate NIB-NTN, GenBank accession number AJ585342) or $\mathrm{PVY}^{\mathrm{N}}$-GFP (PVY N605-GFP [45]) infected plant sap 3 weeks after agroinfiltration with empty TRV vector or mock inoculated as described before $[8,45]$. For experiments without prior agroinfiltration, three plants of each genotype were inoculated 4 weeks after they were transferred to soil. Samples (upper non-inoculated leaves) for RNA isolation (PVY ${ }^{\mathrm{NTN}}$ infected plants) were collected 14 days post inoculation (dpi) and immediately frozen in liquid nitrogen. Samples for confocal microscope observation $\left(\mathrm{PVY}^{\mathrm{N}}\right.$-GFP infected plants) were collected $14 \mathrm{dpi}$ and were stored in humid environment (petri dish with humid paper towel) until observed (maximum time from collection to observation was $4 \mathrm{~h}$ ). For VIGS studies of StWIPK six biological replicates were used and four to six biological replicates (details in Additional file 6) for StMKK6. Upper non-inoculated leaves were sampled at different time points and observed under confocal microscope. For this purpose, leaves were cut and stored in humid environment until observed as described above.

\section{Real-time PCR}

RNA from the samples was isolated with innuPREP Plant RNA Kit and treated with DNAse (Invitrogen, USA; 0.1 $\mathrm{U} /$ Dnase per $\mu \mathrm{g}$ RNA) prior to reverse transcription. $1 \mu \mathrm{g}$ of RNA was reversely transcribed using the High Capacity cDNA Reverse Transcription Kit (Applied Biosystems, USA).

Samples were analyzed in the set-up for quantitative real-time PCR (qPCR) analysis as previously described [57], using TaqMan chemistry for determining the relative concentration of PVY ${ }^{\mathrm{NTN}}$ RNA [58] and cytochrome oxidase (Cox; [59]) as RNA load control. The transcript accumulation was normalized to that of Cox. The relative content of $\mathrm{PVY}^{\mathrm{NTN}}$ was calculated as follows: $\mathrm{Cq}$ value of Cox was subtracted from Cq value for PVY; the resulting value was used as exponential power on value 2 ; every value was finally divided by the minimum value. The resulting value was transformed with base 10 logarithm. Results were statistically evaluated using Two Way 
ANOVA in SigmaPlot 13.0 software. Two independent factors were considered in analysis: Genotype (individual species or clones) and Treatment (only PVY infection or PVY infection with prior agroinfiltration with empty TRV vector). The relative PVY RNA content was the dependent variable. For multiple comparison Tukey's test was selected.

\section{Confocal microscopy}

GFP and background chloroplast fluorescence were visualized with a Leica TCS SP5 laser-scanning microscope mounted on a Leica DMI 6000 CS inverted microscope (Leica Microsystems, Germany) with a HC PL FLUOTAR $10 \times$ objective and with Leica TCS LSI macroscope with Plan APO $1 \times$ or Plan APO $5 \times$ objective (Leica Microsystems, Germany). For excitation, the $488 \mathrm{~nm}$ laser line was used. Fluorescence emissions with wavelengths of 505-530 and 590-680 $\mathrm{nm}$ were collected simultaneously or sequentially through two channels. Images were processed by using Leica LAS AF Lite software (Leica Microsystems, Germany).

\section{Additional files}

Additional file 1. List of Solanum tuberosum cultivars used in this study. All S. tuberosum cultivars used in this study are listed together with the description of its susceptibility to PVY or the genetic change for genetically modified lines.

Additional file 2. Sequence alignment of part of PDS gene originating from different species. Through the alignment, the similarity of the part of PDS gene used in VIGS construct to other plant species is presented.

Additional file 3. List of wild potato relatives used in the studies with their response to TRV-based VIGS. All wild potato relatives used in this study are listed together with their abbreviation clone number, country of origin and silencing response.

Additional file 4. Photographs of mock and PVY NTN infected set of wild potato relatives. One mock inoculated (1) and one PVY $Y^{N T N}$ infected (2) plant at $14 \mathrm{dpi}$ are shown. Letters represent individual species/clones: a) BLB 331-2, b) HJT 349-3, c) JAM 355-1, d) MCQ 186-1, e) OKA 970-3, f) PTA 767-8, g) PLT 378-2, h) SPEC 287-2, i) VNT 250-2, j) VNT 283-1, k) VNT 365-1, I) VNT 366-2, m) VNT 741-1, n) VNT 896-4, o) LES 358-4.

Additional file 5. Report for Two Way ANOVA statistical analysis of relative PVY ${ }^{\text {NTN }}$ RNA content using two independent factors. Statistical comparison (Two Way ANOVA) was conducted between groups of plants from individual clones (genotypes) and groups with different treatment (PVY or PVY + TRV). Difference in PVY NTN RNA content is considered statistically significant, if the $P$ value is lower than 0.05 (all cases are highlighted in yellow).

Additional file 6. Number of infected plants at each time point in StMKK6 silenced and empty vector treated plants for two different experiments. The table shows the actual number of plants, which were used to calculate cumulative percentage of systemically infected plants after TRVbased VIGS in relation to days post inoculation, as shown in Fig. 4.

\section{Authors' contributions}

DD performed the experimental work, acquired the results, carried out the data analysis and interpretation, and wrote the manuscript. AL and TS contributed to studies with StWIPK and StMKK6 and helped in writing the manuscript.
VGAAV, KG and JŽ helped with experimental design and supervised the studies. All authors read and approved the final manuscript.

\section{Author details}

${ }^{1}$ Department of Biotechnology and Systems Biology, National Institute of Biology, Večna Pot 111, 1000 Ljubljana, Slovenia. ${ }^{2}$ Wageningen UR Plant Breeding, Wageningen University and Research Centre, P.O. Box 386, 6700 AJ Wageningen, The Netherlands.

\section{Acknowledgements}

We thank Dr. Richard R.G.F. Visser for providing the collection of wild potato relatives. We also thank Dr. Sabine Rosahl for providing NahG-Désirée potato plants and Dr. Andrej Blejec for help with statistical analysis. We would also like to acknowledge Gerard Bijsterbosch, Juan Du and Maja Jamnik for excellent technical assistance.

\section{Competing interests}

The authors declare that they have no competing interests.

\section{Funding}

The study was supported by the Slovenian Research Agency (Contract Numbers 1000-07-310032, J1-4268 and P4-0165), by COST Action FA0806 and Slovenian Society of Plant Biology.

Received: 3 March 2016 Accepted: 10 May 2016

Published online: 20 May 2016

\section{References}

1. Scholthof K-BG, Adkins S, Czosnek H, Palukaitis P, Jacquot E, Hohn T, Hohn B, Saunders K, Candresse T, Ahlquist P, Hemenway C, Foster GD. Top 10 plant viruses in molecular plant pathology. Mol Plant Pathol. 2011;12:938-54.

2. Whitham SA, Yang C, Goodin MM. Global impact: elucidating plant responses to viral infection. Mol Plant Microbe Interact. 2006;19:1207-15.

3. Kogovšek P, Ravnikar M. Physiology of the potato-potato virus Y interaction. In: Lüttge U, Beyschlag W, Francis D, Cushman J, editors. Progress in Botany SE-3, vol. 74. Berlin: Springer; 2013. p. 101-33.

4. Baebler Š, Stare K, Kovač M, Blejec A, Prezelj N, Stare T, Kogovšek P, Maruša P-N, Rosahl S, Ravnikar M, Gruden K. Dynamics of responses in compatible potato-potato virus $Y$ interaction are modulated by salicylic acid. PLOS ONE. 2011;6:e29009.

5. Gruden K, Pompe-Novak M, Baebler Š, Krečič-Stres H, Toplak N, Hren M, KogovŠek P, Gow L, Foster GD, Boonham N, Ravnikar M. Expression microarrays in plant-virus interaction. Methods Mol Biol. 2008:451:583-613.

6. Kogovšek P, Pompe-Novak M, Baebler Š, Rotter A, Gow L, Gruden K, Foster GD, Boonham N, Ravnikar M. Aggressive and mild potato virus Y isolates trigger different specific responses in susceptible potato plants. Plant Pathol. 2010;59:1121-32.

7. Baebler Š, Witek K, Petek M, Stare K, Tušek-Žnidarič M, Pompe-Novak M, Renaut J, Szajko K, Strzelczyk-Żyta D, Marczewski W, Morgiewicz K, Gruden K, Hennig J. Salicylic acid is an indispensable component of the $\mathrm{Ny}-1$ resistance-gene-mediated response against Potato virus $\mathrm{Y}$ infection in potato. J Exp Bot. 2014;65:1095-109.

8. Baebler S, Krecic-Stres H, Rotter A, Kogovsek P, Cankar K, Kok EJ, Gruden K, Kovac M, Zel J, Pompe-Novak M, Ravnikar M. PVY(NTN) elicits a diverse gene expression response in different potato genotypes in the first $12 \mathrm{~h}$ after inoculation. Mol Plant Pathol. 2009:10:263-75.

9. Baulcombe DC. Fast forward genetics based on virus-induced gene silencing. Curr Opin Plant Biol. 1999;2:109-13.

10. Unver T, Budak H. Virus-induced gene silencing, a post transcriptional gene silencing method. Int J Plant Genomics. 2009;2009:198680.

11. Faivre-Rampant O, Gilroy EM, Hrubikova K, Hein I, Millam S, Loake GJ, Birch P, Taylor M, Lacomme C. Potato virus X-induced gene silencing in leaves and tubers of potato. Plant Physiol. 2004;134:1308-16.

12. Ratcliff F, Martin-Hernandez AM, Baulcombe DC. Technical advance: Tobacco rattle virus as a vector for analysis of gene function by silencing. Plant J. 2008;25:237-45. 
13. Brigneti G, Martín-Hernández AM, Jin H, Chen J, Baulcombe DC, Baker B, Jones JDG. Virus-induced gene silencing in Solanum species. Plant J. 2004;39:264-72

14. Du J, Tian Z, Liu J, Vleeshouwers VGAA, Shi X, Xie C. Functional analysis of potato genes involved in quantitative resistance to Phytophthora infestans. Mol Biol Rep. 2013;40:957-67.

15. Hawkes J. The potato: evolution, biodiversity and genetic resources London: Belhaven Press; 1990.

16. Spooner DM, Berg RG van den, Rodrigues A, Bamberg JB, Hijmans RJ, Lara-Cabrera S. Wild potatoes (Solanum section Petota; Solanaceae) of North and Central America. BIS, Leerstoelgroep Biosystematiek, 30: The American Society of Plant Taxonomists; 2004 (Systematic Botany Monographs: 68).

17. Berloo R, Hutten RCB, Eck HJ, Visser RGF. An online potato pedigree database resource. Potato Res. 2007;50:45-57.

18. Jacobs MM, van den Berg RG, Vleeshouwers VG, Visser M, Mank R, Sengers M, Hoekstra R, Vosman B. AFLP analysis reveals a lack of phylogenetic structure within Solanum section Petota. BMC Evol Biol. 2008:8:145.

19. Vleeshouwers VGAA, Finkers R, Budding D, Visser M, Jacobs MMJ, van Berloo R, Pel M, Champouret N, Bakker E, Krenek P, Rietman H, Huigen D, Hoekstra R, Goverse A, Vosman B, Jacobsen E, Visser RGF. SolRgene: an online database to explore disease resistance genes in tuber-bearing Solanum species. BMC Plant Biol. 2011;11:116.

20. Ishihama N, Yamada R, Yoshioka M, Katou S, Yoshioka H. Phosphorylation of the Nicotiana benthamiana WRKY8 transcription factor by MAPK functions in the defense response. Plant Cell. 2011;23:1153-70.

21. Mase K, Mizuno T, Ishihama N, Fujii T, Mori H, Kodama M, Yoshioka H. Ethylene signaling pathway and MAPK cascades are required for AAL Toxin-induced programmed cell death. Mol Plant Microbe Interact. 2012;25:1015-25

22. Samuel MA, Hall H, Krzymowska M, Drzewiecka K, Hennig J, Ellis BE. SIPK signaling controls multiple components of harpin-induced cell death in tobacco. Plant J. 2005:42:406-16.

23. Seo S, Katou S, Seto H, Gomi K, Ohashi Y. The mitogen-activated protein kinases WIPK and SIPK regulate the levels of jasmonic and salicylic acids in wounded tobacco plants. Plant J. 2007;49:899-909.

24. Yap Y, Kodama Y, Waller F, Chung KM, Ueda H, Nakamura K, Oldsen M, Yoda H, Yamaguchi Y, Sano H. Activation of a novel transcription factor through phosphorylation by WIPK, a wound-induced mitogen activated protein kinase in tobacco plants. Plant Physiol. 2005:139:127-37.

25. Ahlfors R, Macioszek V, Rudd J, Brosché M, Schlichting R, Scheel D, Kangasjärvi J. Stress hormone-independent activation and nuclear translocation of mitogen-activated protein kinases in Arabidopsis thaliana during ozone exposure. Plant J. 2004:40:512-22.

26. Kovtun Y, Chiu WL, Tena G, Sheen J. Functional analysis of oxidative stressactivated mitogen-activated protein kinase cascade in plants. Proc Natl Acad Sci USA. 2000;97:2940-5.

27. Gudesblat GE, lusem ND, Morris PC. Guard cell-specific inhibition of Arabidopsis MPK3 expression causes abnormal stomatal responses to abscisic acid and hydrogen peroxide. New Phytol. 2007;173:713-21.

28. Hamel L-PP, Nicole M-CC, Sritubtim S, Morency M-JJ, Ellis M, Ehlting J, Beaudoin N, Barbazuk B, Klessig D, Lee J, Martin G, Mundy J, Ohashi Y, Scheel D, Sheen J, Xing T, Zhang S, Seguin A, Ellis BE. Ancient signals: comparative genomics of plant MAPK and MAPKK gene families. Trends Plant Sci. 2006:11:192-8.

29. Yang Q, Hua J, Wang L, Xu B, Zhang H, Ye N, Zhang Z, Yu D, Cooke HJ, Zhang Y, Shi Q. MicroRNA and piRNA profiles in normal human testis detected by next generation sequencing. PLoS ONE. 2013;8:e66809.

30. Wang H, Ngwenyama N, Liu Y, Walker JC, Zhang S. Stomatal development and patterning are regulated by environmentally responsive mitogenactivated protein kinases in Arabidopsis. Plant Cell. 2007;19:63-73.

31. García-Marcos A, Pacheco R, Martiáñez J, González-Jara P, Díaz-Ruíz JR, Tenllado F. Transcriptional changes and oxidative stress associated with the synergistic interaction between potato virus $X$ and potato virus $Y$ and their relationship with symptom expression. Mol Plant Microbe Interact. 2009:22:1431-44.

32. Hardin SC, Wolniak SM. Molecular cloning and characterization of maize ZmMEK1, a protein kinase with a catalytic domain homologous to mitogen- and stress-activated protein kinase kinases. Planta. 1998;206:577-84.
33. Hardin SC, Wolniak SM. Expression of the mitogen-activated protein kinase kinase ZmMEK1 in the primary root of maize. Planta. 2001:213:916-26.

34. Soyano T, Nishihama R, Morikiyo K, Ishikawa M, Machida Y. NQK1/NtMEK1 is a MAPKK that acts in the NPK1 MAPKKK-mediated MAPK cascade and is required for plant cytokinesis. Genes Dev. 2003;17:1055-67.

35. Takahashi Y, Soyano T, Kosetsu K, Sasabe M, MacHida Y. HINKEL kinesin, ANP MAPKKKs and MKK6/ANQ MAPKK, which phosphorylates and activates MPK4 MAPK, constitute a pathway that is required for cytokinesis in Arabidopsis thaliana. Plant Cell Physiol. 2010;51:1766-76.

36. Liu YY, Zhou Y, Liu L, Sun L, Zhang M, Liu YY, Li D. Maize ZmMEK1 is a single-copy gene. Mol Biol Rep. 2012;39:2957-66.

37. Lazar A, Coll A, Dobnik D, Baebler S, Bedina-Zavec A, Zel J, Gruden K. Involvement of potato (Solanum tuberosum L.) MKK6 in response to potato virus Y. PLOS ONE. 2014;9:e104553.

38. Jin H, Liu Y, Yang K-YY, Kim CY, Baker B, Zhang S. Function of a mitogenactivated protein kinase pathway in $\mathrm{N}$ gene-mediated resistance in tobacco. Plant J. 2003:33:719-31.

39. Liu Y, Schiff M, Dinesh-Kumar SP. Involvement of MEK1 MAPKK, NTF6 MAPK, WRKY/MYB transcription factors, COl1 and CTR1 in N-mediated resistance to tobacco mosaic virus. Plant J. 2004;38:800-9.

40. Liu Y, Ren D, Pike S, Pallardy S, Gassmann W, Zhang S. Chloroplast-generated reactive oxygen species are involved in hypersensitive response-like cell death mediated by a mitogen-activated protein kinase cascade. Plant J. 2007:51:941-54

41. Halim VA, Hunger A, Macioszek V, Landgraf P, Nürnberger T, Scheel D, Rosahl S. The oligopeptide elicitor Pep-13 induces salicylic acid-dependent and -independent defense reactions in potato. Physiol Mol Plant Pathol. 2004;64:311-8.

42. Dobnik D, Baebler S, Kogovšek P, Pompe-Novak M, Stebih D, Panter G, Janež N, Morisset D, Zel J, Gruden K. ß-1,3-Glucanase class III promotes spread of PVY(NTN) and improves in planta protein production. Plant Biotechnol Rep. 2013:7:547-55.

43. Demmig-Adams B, Adams WW. Photoprotection and other responses of plants to high light stress. Annu Rev Plant Physiol Plant Mol Biol. 1992;43:599-626.

44. Pompe-Novak M, Gruden K, Baebler Š, Krečič-Stres H, Kovač M, Jongsma M, Ravnikar M. Potato virus $Y$ induced changes in the gene expression of potato (Solanum tuberosum L.). Physiol Mol Plant Pathol. 2006;67:237-47.

45. Rupar M, Faurez F, Tribodet M, Gutiérrez-Aguirre I, Delaunay A, Glais L, Kriznik M, Dobnik D, Gruden K, Jacquot E, Ravnikar M. Fluorescently tagged potato virus Y: a versatile tool for functional analysis of plant-virus interactions. Mol Plant Microbe Interact. 2015;28:739-50.

46. Zhang S, Klessig DF. Resistance gene N-mediated de novo synthesis and activation of a tobacco mitogen-activated protein kinase by tobacco mosaic virus infection. Proc Natl Acad Sci USA. 1998;95:7433-8.

47. Zhang S, Klessig DF. The tobacco wounding-activated mitogenactivated protein kinase is encoded by SIPK. Proc Natl Acad Sci USA. 1998:95:7225-30.

48. Kishi-Kaboshi M, Okada K, Kurimoto L, Murakami S, Umezawa T, Shibuya N, Yamane H, Miyao A, Takatsuji H, Takahashi A, Hirochika H. A rice fungal MAMP-responsive MAPK cascade regulates metabolic flow to antimicrobial metabolite synthesis. Plant J. 2010:63:599-612.

49. Melech-Bonfil S, Sessa G. Tomato MAPKKKE is a positive regulator of cell-death signaling networks associated with plant immunity. Plant J. 2010;64:379-91.

50. Liu Y, Jin H, Yang K-Y, Kim CY, Baker B, Zhang S. Interaction between two mitogen-activated protein kinases during tobacco defense signaling. Plant J. 2003;34:149-60.

51. Alazem M, Lin N-S. Roles of plant hormones in the regulation of hostvirus interactions. Mol Plant Pathol. 2015;16:529-40.

52. Brodersen P, Petersen M, Bjørn Nielsen H, Zhu S, Newman M-A, Shokat KM, Rietz S, Parker J, Mundy J. Arabidopsis MAP kinase 4 regulates salicylic acid- and jasmonic acid/ethylene-dependent responses via EDS1 and PAD4. Plant J. 2006;47:532-46.

53. Petersen $M$, Brodersen $P$, Naested $H$, Andreasson $E$, Lindhart $U$, Johansen B, Nielsen HB, Lacy M, Austin MJ, Parker JE, Sharma SB, Klessig DF, Martienssen R, Mattsson O, Jensen AB, Mundy J. Arabidopsis map kinase 4 negatively regulates systemic acquired resistance. Cell. 2000;103:1111-20. 
54. del Pozo O, Pedley KF, Martin GB. MAPKKKalpha is a positive regulator of cell death associated with both plant immunity and disease. EMBO J. 2004;23:3072-82.

55. Liu Y, Schiff M, Dinesh-Kumar SP. Virus-induced gene silencing in tomato. Plant J. 2002;31:777-86.

56. Du J, Rietman H, Vleeshouwers VGAA. Agroinfiltration and PVX agroinfection in potato and Nicotiana benthamiana. J Vis Exp. 2014;83:e50971.

57. Hren M, Nikolić P, Rotter A, Blejec A, Terrier N, Ravnikar M, Dermastia M, Gruden K. "Bois noir" phytoplasma induces significant reprogramming of the leaf transcriptome in the field grown grapevine. BMC Genom. 2009;10:460.
58. Kogovšek P, Gow L, Pompe-Novak M, Gruden K, Foster GD, Boonham N, Ravnikar M. Single-step RT real-time PCR for sensitive detection and discrimination of potato virus Y isolates. J Virol Methods. 2008;149:1-11.

59. Weller SA, Elphinstone JG, Smith NC, Boonham N, Stead DE. Detection of Ralstonia solanacearum strains with a quantitative, multiplex, real-time, fluorogenic PCR (TaqMan) assay. Appl Environ Microbiol. 2000;66:2853-8.

\section{Submit your next manuscript to BioMed Central and we will help you at every step:}

- We accept pre-submission inquiries

- Our selector tool helps you to find the most relevant journal

- We provide round the clock customer support

- Convenient online submission

- Thorough peer review

- Inclusion in PubMed and all major indexing services

- Maximum visibility for your research

Submit your manuscript at

www.biomedcentral.com/submit 